



\title{
¿Agua si, oro no? Anclajes del extractivismo y el ambientalismo en el páramo de Santurbán ${ }^{1}$
}

\author{
Alejandra Osejo ${ }^{2}$ \\ Instituto Alexander von Humboldt, Colombia ${ }^{3}$ \\ aosejo@humboldt.org.co \\ Paula Ungar ${ }^{4}$ \\ Rachel Carson Center for Environment and Society, Alemania ${ }^{5}$ \\ paula.ungar@gmail.com
}

Recibido: 30 de julio de 2016

Aceptado: 15 de febrero de 2017

Disponible en linea: 14 de diciembre de 2017

\footnotetext{
1 Este artículo es producto de la investigación sobre actores sociales en el páramo de Santurbán desarrollada por las autoras en el Instituto Humboldt. Agradecemos a las entidades financiadoras (Unión Europea y Ministerio de Ambiente y Desarrollo Sostenible) y a otros investigadores que aportaron en el proceso: Camilo Sarmiento, Rigoberto Abello, Laura Roldán, Susana Peláez y Emerson Buitrago. La fase final de elaboración del manuscrito fue posible gracias a una beca del Rachel Carson Center for Environment and Society (RCC) (Munich, Alemania), que le fue concedida a la segunda autora.

2 Magíster en Desarrollo Rural, Universidad Federal Rio Grande do Sul (Brasil).

3 Investigadora Adjunta, Programa de Ciencias Sociales y Saberes de la Biodiversidad (Bogotá, Colombia).

4 Ph.D., Universitat Autónoma de Barcelona (España).

5 Writing Fellow 2016-2017.
} 


\title{
¿Agua si, oro no? Anclajes del extractivismo y el ambientalismo en el páramo de Santurbán
}

\section{Resumen}

En los últimos años, los páramos han adquirido protagonismo en el escenario público colombiano como territorios disputados entre diferentes intereses, visiblemente entre aquellos alineados en torno al cuidado del agua y los vinculados al extractivismo minero. A través de un estudio de caso en el páramo de Santurbán, una zona clave para la provisión de agua para más de un millón de habitantes urbanos y rurales de sus alrededores, que alberga importantes reservas de oro y actividad agrícola intensiva, este trabajo aporta a la comprensión de los páramos como territorios cuyas transformaciones resultan de complejas interacciones históricas e interescalares, visibles tanto en torno al extractivismo como al ambientalismo. Apoyándonos en una perspectiva relacional y en el concepto de fricción propuesto por Anna Tsing, pretendemos aportar a la comprensión de esta complejidad y llamar la atención sobre la necesidad de tener en cuenta la multiescalaridad en las políticas de Estado con objetivos conservacionistas.

Palabras clave: páramos; global-local; conflictos socioambientales; conservación; análisis relacional; etnografia multisituada; política de la escala

\section{Yes to Water, No to Gold? Connections of Extractivism and Environmentalism in the Santurbán Páramo}

\begin{abstract}
In recent years, equatorial upper mountain ecosystems known as páramos have gained public prominence in Colombia as territories disputed among different interests, noticeably among those lined up around water care and those related to mining extractivism. Through a case study on the Santurbán Páramo, a key water supply for more than one million urban and rural inhabitants of its surroundings, which hosts important gold reserves and intensive farming, this work contributes to understanding moorlands as territories whose transformations occur as a result of complex historical and inter-scale interactions, visible around both extractivism and environmentalism. Based on a relational perspective and on the concept of friction proposed by Anna Tsing, this work intends to contribute to the understanding of this complexity and draw attention to the need for considering the multiscalar in governmental policies with conservation purposes.
\end{abstract}

Keywords: páramo; global-local; socio-environmental conflicts; conservation; relational analysis; multi-sited ethnography; politics of scale

\section{Água sim, ouro não? Ancoragens do extrativismo e o ambientalismo no Páramo de Santurbán}

\section{Resumo}

Nos últimos anos, os páramos adquiriram protagonismo na arena pública colombiana como territórios disputados entre diferentes interesses, visivelmente entre aqueles alinhados em torno do cuidado da agua e os vinculados ao extrativismo de mineração. Através de um estudo de caso no páramo de Santurbán, área chave para a provisão de agua de mais de um milhão de habitantes urbanos e rurais dos redores, que abriga importantes reservas de ouro e atividade agrícola intensiva, este trabalho aporta na compreensão dos páramos como territórios cujas transformações resultam de complexas interações históricas e interescalares, visíveis tanto em torno do extrativismo quanto do ambientalismo. Baseados em uma perspectiva relacional e no conceito de Fricção proposto por Anna Tsing, visamos contribuir na compreensão de tal complexidade e chamar a atenção sobre a necessidade de levar em conta a multiescalaridade nas politicas de Estado com objetivos conservacionistas. Palavras-chave: páramos; global-local; conflitos socioambientais; conservação; análise relacional; etnografia multisituada; política da escala 


\section{Introducción}

En una visita a Colombia en abril de 2014, Al Gore, exvicepresidente de Estados Unidos y premio Nobel, afirmó que "Colombia tiene que escoger entre el oro en los páramos y ganancias para unos cuantos, o el agua potable que abastece a todos sus ciudadanos". Esta afirmación fue hecha en medio de acalorados debates públicos que se dispararon desde que en 2009 la minera canadiense Greystar solicitara una licencia para minería de oro a cielo abierto en Santurbán, en la parte alta de la cuenca que abastece de agua a la ciudad de Bucaramanga.

En este artículo pretendemos argumentar que reducir las tensiones en torno al páramo a un problema binario, en el cual el extractivismo, de un lado, y el ambientalismo, del otro, son fuerzas independientes y opuestas que se despliegan en un escenario pasivo, sin historia, constituye una lectura equivocada de la situación.

La reflexión que presentamos aquí se construyó con base en un análisis de actores sociales vinculados a Santurbán, elaborado por las autoras como insumo para la formulación de recomendaciones de politica. ${ }^{6} \mathrm{~A}$ medida que avanzábamos en esa tarea, se hacían visibles los vínculos complejos entre escalas, ${ }^{7}$ en particular entre los actores con incidencia global y los sucesos en el territorio, tanto en torno al extractivismo como a la conservación. Al relacionarse con la historia particular de este páramo, estas fuerzas globales, aunque efectivamente demuestran su agencia, también producen alianzas y tensiones inesperadas.

El papel de lo global en los conflictos ambientales ha sido objeto de reflexión desde diferentes disciplinas y con diferentes énfasis. La interacción entre escalas espaciales y temporales ha sido central para la comprensión de los problemas ambientales en términos políticos por lo menos desde la década de 1980 (véase, por

6 Los resultados completos se encuentran en Ungar, Osejo et al. (2014).

7 En este artículo usamos 'escala' para referirnos tanto a las dimensiones del análisis (escala geográfica, escala temporal, por ejemplo) como a las unidades de medida al interior de estas dimensiones (local, regional, nacional, por ejemplo). 
ejemplo, Blaikie y Brookfield, 1987). Dos décadas después, la ecología política se inclina por entender la politica de la escala; la construcción social y la contingencia de las escalas sociales-ecológicas (Zimmerer, 2006; Neumann, 2009; Sletto, 2009); y la escala como resultado de ejercicios de poder y manifestación de proyectos políticos (Tsing, 2005). En este contexto, el estudio de las redes - la producción de espacialidades relacionales como eje de los conflictos en torno al control del territorio- se ha convertido en un tema central (por ejemplo, Swyngedouw, 2004).

En la ecología política latinoamericana, la mirada a esta relación entre escalas ha tendido a centrarse en las asimetrias, los enfrentamientos entre lo local/el lugar y lo global; en las resistencias populares frente al comercio ecológicamente desigual y la imposición de algunos lenguajes de valoración sobre otros (Martínez-Alier, 2005); en la posición subordinada de los pueblos colonizados (Alimonda, 2011); en las alianzas entre Estados y multinacionales extractivistas en torno al "consenso de los Commodities" enfrentado a la defensa del territorio alrededor del "giro ecoterritorial" (Svampa, 2012). Con un énfasis más epistemológico, recogiendo tradiciones latinoamericanas de pensamiento, Arturo Escobar, desde el proyecto modernidad/colonialidad, se centra en la crítica a los modos eurocéntricos de entender la modernidad e invita a enfocar el pensamiento en las márgenes, en mundos alternativos (Escobar, 2003), y a entender, desde la categoría analítica lugar, la experiencia de las localidades, sus grados de enraizamiento, linderos y conexión con la vida diaria como formas alternativas de construir el mundo.

El concepto de fricción propuesto por Anna Tsing (2005), a la vez que recoge puntos críticos de varias de estas líneas de pensamiento, ofrece un marco de análisis que coincide con nuestro interés en mirar el encuentro de lo global con el lugar (en vez de lo local en tanto inmerso en una globalidad imperante) y resuena con nuestro trabajo metodológico, que se caracteriza por ser relacional y etnográfico. La fricción, según esta autora, es una forma de enmarcar los encuentros entre los universales, que circulan en lo global, y las particularidades de su expresión concreta, de su anclaje en el territorio. Tsing se distancia 
de una comprensión de lo global como una unidad homogénea que se despliega sin tropiezos sobre un mundo de localidades diversas, y prefiere enfocarse en las formas en las que "lo global se escenifica a través de compromisos y prácticas" (Tsing, 2005, p. 9). Esta autora se pregunta "¿cómo es que el conjuro de lo global logra afectar-explotar-incidir en mundos geográfica, cultural y políticamente remotos?”, y responde, apoyándose en la idea de la política de la escala, que "es a través de articulaciones contingentes en las cuales el conjuro financiero globalista se vincula a otros proyectos nacionales y regionales de producción de escalas (conjuros)". Parafraseándola, nos preguntamos entonces cómo es que los universales del ambientalismo y el extractivismo se hacen efectivos dentro de la coyuntura particular de Santurbán entre 2012 y 2014, y cómo se desenvuelve la contingencia de los ejercicios de poder en este territorio.

En este trabajo entendemos por extractivismo, en consonancia con Göbel y Ulloa (2014), un modo de producción basado en la explotación de recursos a gran escala demandados globalmente, generalmente orientado a la exportación, y que conlleva la instalación de un enclave transnacional en áreas periféricas. Por ambientalismo entendemos el conjunto de diversas expresiones politicas de individuos y grupos sociales donde el sujeto de debate es el ambiente y su relación con la vida humana. Ya en la década de 1990, Gudynas (1992) reconocía como centro de estas preocupaciones cuestiones como la conservación y el manejo de los ecosistemas, el impacto de actividades extractivas y el comercio internacional, entre otros. En ese momento caracterizó el ambientalismo latinoamericano por su contenido utopista que critica la idea del crecimiento económico como motor del progreso social. También reconoció la heterogeneidad interna y la permeabilidad externa de este tipo de movimientos sociales y resaltó los retos posteriores a la conferencia de Río de Janeiro, cuando el discurso ambiental fue incorporado por otros actores como empresarios, industriales y diversos tipos de estados.

Este enfoque, propuesto desde hace varias décadas, evidencia que no hay unanimidad en las reivindicaciones de quienes confluyen en la defensa de la naturaleza. Los enfoques más recientes 
mencionados llaman la atención acerca de la necesidad de comprender esta heterogeneidad y sus fricciones desde una perspectiva escalar. Proponemos entonces analizar, en el caso específico de Santurbán, los encuentros y tensiones entre el ambientalismo y el extractivismo.

\section{Metodologia}

En este artículo reflexionamos sobre los resultados de una aproximación multiescalar y multitemporal a los actores sociales vinculados a Santurbán. Siguiendo la invitación de Del Cairo et al. (2015), esta aproximación nos permite considerar las complejas interacciones entre escalas geográficas, politicas y temporales para el entendimiento de los conflictos socioambientales. La perspectiva multitemporal insiste en la necesidad de incorporar la transformación de las relaciones entre sociedades y naturaleza en el tiempo, y las formas en las que estas dinámicas exigen ajustes en las relaciones de poder.

La caracterización de los actores y de sus relaciones entre sí y con el páramo se desarrolló desde una perspectiva relacional, vinculando algunos de los instrumentos que ofrece el Análisis de Redes Sociales (ARS) (Palacio, 2015) y procedimientos cualitativos como la etnografia multisituada (Marcus, 2001; Falzon, 2012). El ARS se ha usado para entender las relaciones en torno a la gobernanza ambiental (Bodin y Crona, 2009), y en Colombia se ha aplicado para hacer aportes a la gestión ambiental territorial (Palacio y Hurtado, 2005; Palacio y van der Hammen, 2007).

A través de la recolección y sistematización de fuentes secundarias y primarias, se identificaron y caracterizaron $\operatorname{los}_{\text {actores }}^{8}$ involucrados en el uso, la conservación y el manejo del Complejo de Páramos Jurisdicciones Santurbán - Berlín (CPJSB) en el periodo comprendido

Definimos los actores como grupos formalmente constituidos, involucrados en el uso, manejo y conservación del CPJSB a diferentes niveles en el periodo estudiado. 
entre diciembre de 2009 y diciembre de $2012,{ }^{9}$ con acciones a diferentes escalas, y se clasificaron los vínculos entre ellos, en términos generales, como "de afinidad" o "de conflicto". Esto nos permitió determinar la composición y estructura de las redes. ${ }^{10}$ Una caracterización a mayor profundidad de actores y vínculos se desarrolló con base en trabajo etnográfico realizado en el mismo periodo. Además, se sistematizó la información cronológica encontrada en las fuentes secundarias en una línea de tiempo, en diálogo con los resultados etnográficos, y los eventos se clasificaron en categorias relacionadas con las transformaciones del territorio, los procesos demográficos, las dinámicas de organización, gobierno y control y los procesos productivos y extractivos.

\section{El territorio diferenciado de Santurbán}

El CPJSB se extiende sobre 142.608 hectáreas del extremo norte de la cordillera Oriental colombiana, en jurisdicción de 30 municipios de Santander y Norte de Santander (Sarmiento y Ungar, 2014). E1 agua que proviene de este complejo drena hacia las cuencas del Magdalena, por el occidente, y del Catatumbo, por el oriente, a través de ocho subzonas hidrográficas de importancia regional.

Nuestro análisis nos permitió, en primer lugar, entender el páramo como diferenciable en tres unidades, que podrian denominarse socioecológicas, en la medida en que encontramos entre ellas notables diferencias biofisicas en cuanto a los beneficiarios de sus fuentes de agua, en su historia ambiental y en los actores y los vínculos entre ellos y con el territorio. A estas unidades haremos referencia de aquí en adelante.

Se tomó ese hito inicial debido a que en ese mes la compañía Greystar solicitó licencia ambiental para minería a cielo abierto - Proyecto Angosturas (California) - lo cual desencadenó (o hizo visibles) diferentes grupos e iniciativas del Estado y de la sociedad en torno a la gestión del páramo.

${ }^{10}$ Los resultados gráficos del análisis elaborado se pueden consultar en Ungar, Osejo et al. (2014). Para la argumentación en la que pretendemos hacer énfasis en este artículo, y por consideraciones de extensión, no juzgamos pertinente presentar aquí las gráficas asociadas. Sin embargo, esta argumentación se construyó describiendo y analizando los vínculos allí encontrados. 


\section{Articulaciones del proyecto extractivista global}

La unidad occidental de Santurbán comprende las zonas altas de ocho municipios del departamento de Santander, entre los cuales están Vetas y California, y aunque no es la unidad más habitada sí incluye un casco urbano en el páramo. El río Suratá, que nace en esta unidad, abastece parte del sistema del Acueducto Metropolitano de Bucaramanga (AMB), el cual surte a más de 1'300.000 habitantes del área metropolitana. Del agua de esta vertiente también se abastecen las cabeceras de Vetas, Suratá, Charta y California, y numerosos acueductos veredales.

Nuestro estudio identificó una gran cantidad y diversidad de actores y vínculos en torno a esta unidad, especialmente conectados con la actividad minera (empresas, sindicatos, entidades del estado encargadas de su regulación), de una parte, y con la organización civil de diferentes tipos y niveles (gremios, partidos politicos y organizaciones locales, regionales y nacionales), de otra. También fue notable la gran incidencia de actores con anclaje global, vinculados a actores de las escalas nacional, regional y local, así como las tensiones con organizaciones asociadas a reivindicaciones ambientalistas.

En el periodo estudiado, las empresas mineras transnacionales con interés en desarrollar proyectos de exploración y explotación de oro determinaban en gran parte la dinámica productiva y laboral de la zona. Según Zapata (2012), las principales empresas con incidencia en la zona eran CB Gold, ${ }^{11}$ AUX, ${ }^{12}$ White Gold Corporation, ${ }^{13}$ Galway Resourses ${ }^{14}$ y EcoOro. Esta última es una empresa canadiense dedicada a la exploración de metales preciosos con títulos mineros en Tona, Charta, Vetas y

11 Desde el 2009, esta empresa canadiense había comprado 12 títulos mineros en Vetas, de los cuales 6 estaban a su nombre, ubicados entre los 2.800 y $4.200 \mathrm{msnm}$. Empleaba a 117 personas y esperaba producir entre 80.000 y 100.000 onzas de oro anuales.

12 Esta empresa pertenece al grupo empresarial brasilero EBX. Este ha comprado títulos en Vetas, Suratá y California a Ventana Gold, que tenía presencia desde el 2005 en el municipio de California. En el 2012, Qatar Investment Found compró el $49 \%$ de AUX.

${ }^{13}$ Empresa canadiense dedicada a la exploración de oro a través de su filial Oro Barracuda. En 2012 tenía seis títulos mineros y cinco solicitudes en el municipio de Suratá.

14 Galway Resources tenía en el momento del estudio de Zapata dos proyectos en la zona: uno en Vetas y otro en California. En Vetas, la empresa firmó un acuerdo vinculante para adquirir los derechos y propiedades de la empresa Reina de Oro, que a su vez había comprado en 1969 los derechos mineros a Benjamin Breewer. En el 2007 esta empresa familiar firmó un contrato de arrendamiento con opción de compra con White Gold Corporation y Oro Barracuda, que realizó la exploración. 
California. Esta empresa está en la zona, bajo el nombre Greystar, desde 1994, cuando comenzó a adquirir derechos mineros de particulares y terrenos en California, Suratá y Vetas. En 2009, Greystar solicitó licencia para la explotación a cielo abierto de Angostura.

El progresivo control de esta zona por parte de multinacionales mineras desde la década de 1990 es el resultado de un proceso histórico que se remonta hasta la Conquista; desde entonces, Santurbán ha estado inserta en proyectos económicos y políticos transnacionales. Es posible hacer esta lectura histórica a través de tres momentos: la minería en la Conquista y la Colonia, la promoción de la minería en la República y la cooperación internacional en torno al mejoramiento tecnológico entre 1998 y 2008.

En 1532, el alemán Ambrosio de Alfinger, gobernador de la provincia de Coro (hoy Venezuela), en representación de la compañía alemana Bezares (Bartolomé Welser y Compañia ${ }^{15}$ ), ingresa por el oriente a territorio Guane y llega al actual valle de Chinácota cruzando los páramos. Diecisiete años después se funda la ciudad de Pamplona, en gran parte gracias a la riqueza proveniente de las minas de oro — sería conocida como "Pamplonita la loca" por cuenta de la ostentación del oro-, y a los pocos años surgen, en territorios habitados por indígenas chitareros, los reales de minas en zonas de páramo denominados La Montuosa Alta, Vetas y Páramo Rico. Estas minas, instauradas en las cercanías de los principales cursos de agua, permitieron el establecimiento permanente de los españoles y de enclaves para el control del territorio. Fueron explotadas por medio del sistema de mita con indigenas de las comunidades chitarero, lache-u'wa y muisca (Riaño, 2015), pertenecientes a los encomenderos de Pamplona y Tunja. El auge de la minería de oro entre 1550 y 1650 requirió del cultivo de grandes cantidades de trigo, cebada y otros productos en los valles del sur del complejo, en los municipios de Tona, Suratá y Cácota, para el suministro de alimentos para los mineros, lo que implicó la transformación de grandes zonas de

\footnotetext{
${ }^{15}$ Los Welser eran una familia de banqueros de Augsburgo y una de las principales casas financieras de Europa en la primera mitad del siglo XVI, dedicada a la explotación de minas de plata en Europa central, entre otras actividades. En 1525, Carlos I abre la posibilidad de que extranjeros se establezcan en América en las mismas condiciones que los españoles. Bartolome Welser consigue, como parte de pago de una deuda, que se le otorgue el derecho a adelantar la conquista y colonización de la Provincia de Venezuela.
} 
lo que hoy se conoce como Berlín (Riaño, 2015). Berlín es la unidad más seca y plana de Santurbán -más fácilmente cultivable-, y aún hoy la más transformada para fines agrícolas, con casi un $45 \%$ de modificación de sus coberturas (véase la figura 1).

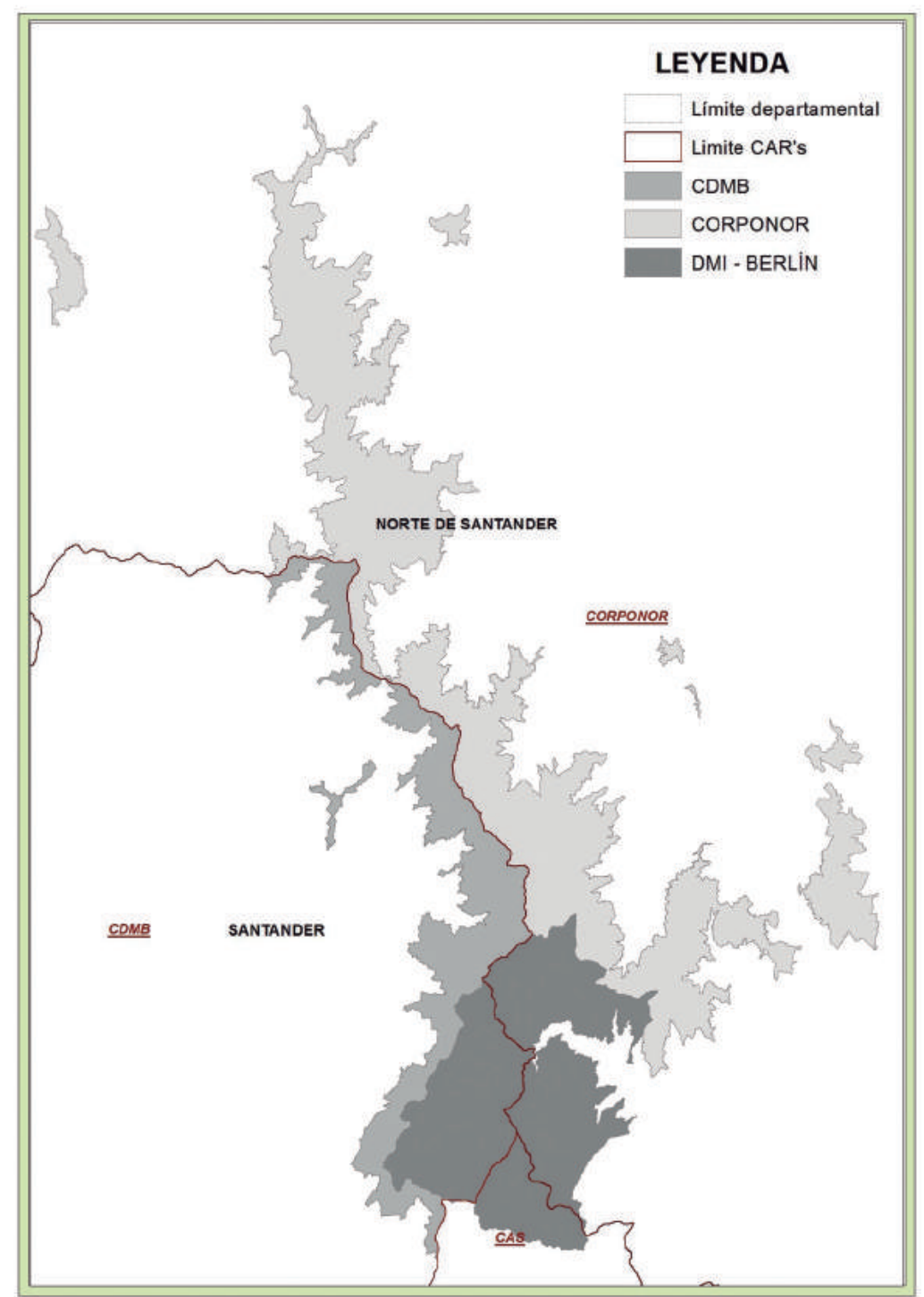

Figura 1. Unidades al interior del complejo de páramos CPJSB. Fuente: Sarmiento, C. y Ungar, P. (Eds.). (2014). 
Es así como el oro se convierte en el material de anclaje del proyecto colonial expansionista y en medio para la consolidación del esquema de control social y tenencia y uso de la tierra en el norte del Virreinato de la Nueva Granada.

Casi trescientos años más tarde todavía es posible ver vínculos de empresas de capital transnacional en Santurbán, a través del oro. En 1830, la compañía inglesa Colombian Mining Association inicia sus labores en la Real de Minas de Muntuosa la Baja, ubicada en el actual municipio de California, instalando dos molinos para la extracción de plata y oro. La presencia de esta empresa en la zona obedece al interés más amplio de los británicos de apoderarse de yacimientos mineros en la Nueva Granada a través de sus casas comerciales. Por su parte, el gobierno colombiano dispuso la exención de impuesto a los instrumentos empleados en la minería, la exención de servicio militar a los mineros, y la creación de un museo y una escuela minera (Gil Granada, 2010). Posteriormente, en 1901, la compañía francesa Francia Gold and Silver Limited inicia el proyecto Llano Redondo en la misma zona.

Como ha sucedido hasta la actualidad, proyectos europeos interoceánicos de construcción de escala se conectan con proyectos de control político americano, fortaleciéndose mutuamente.

Más adelante, en la segunda mitad del siglo XX, se conforman sociedades familiares en torno a la extracción de oro. Es el caso de la familia Moreno, que en 1969 crea la empresa Reina de Oro, y de las empresas Elsy y Providencia, que inician actividades en 1970. En 2012, existían en Santurbán cuatro empresas familiares: El Volcán, Trompetero, La Providencia y La Elsy (Buitrago, 2012). Esta dinámica histórica en la zona explica en gran medida las configuraciones actuales de la población de Vetas y California, que se autoidentifica como minera, y señala la relación intima entre su actividad, el agua y el páramo. Estas empresas familiares, sin embargo, han sido progresivamente adquiridas por multinacionales a partir del final del siglo XX, lo que ha transformado considerablemente la extracción del oro y las relaciones de los actores asociados. 


\section{Encadenamientos del ambientalismo y movimientos sociales}

La llegada renovada del extractivismo minero al occidente de Santurbán a partir de la década de 1990, catalizó una serie de eventos, a través de lo que Tsing llamaría insurrección. En particular, como reacción a la solicitud de mina a cielo abierto por parte de Greystar en 2009, se consolidó una poderosa red regional en torno al ambientalismo, con centro en Bucaramanga y con vínculos efectivos con actores que se desenvuelven en lo nacional y lo global.

Sin embargo, la conexión entre escalas y la participación de lo global en Santurbán en torno al medio ambiente no es un evento aislado. Al menos desde la década de 1990, los efectos de la minería en la calidad del agua habían preocupado a las autoridades ambientales regionales, una vez se identificó que los deshechos contaminados con mercurio y cianuro eran vertidos en el río Suratá, muy cerca de la planta de tratamiento Bosconia, que abastece a parte de la población de Bucaramanga. ${ }^{16}$ Se dio así una relación de colaboración entre dicha autoridad, las empresas familiares y la cooperación internacional alemana en la búsqueda de tecnologías para disminuir la contaminación por mercurio. Expresión de dicha relación es la asociación entre la Corporación Autónoma Regional para la Defensa de la Meseta de Bucaramanga (CDMB), la Compañía de Acueducto Metropolitano de Bucaramanga (CAMB), la Gobernación de Santander y las autoridades mineras en torno al proyecto Reducción de la contaminación ambiental debida a la pequeña minería en la cuenca del río Suratá (1997-2008). Este convenio de cooperación técnica aportó a la reducción del uso de mercurio y cianuro (Zapata, 2012) y contribuyó a construir lazos de confianza entre las empresas familiares y la autoridad ambiental (Buitrago, 2012). Al finalizar la financiación proveniente de la cooperación alemana, el proyecto fue abandonado paulatinamente.

\footnotetext{
${ }^{16}$ El diagnóstico que sustentó el desarrollo del proyecto encontró 11 plantas activas en Vetas y 14 en California, las cuales producían entre 250 y 350 kilogramos de oro anualmente. También calculó que, de los 3.500 habitantes estimados de la región, un $90 \%$ dependía directa o indirectamente de la minería como sustento. En el monitoreo realizado por la CDMB en algunos puntos de la corriente superficial (inmediatamente después de las zonas con actividad minera) del río Vetas y la quebrada La Baja se corroboró la presencia de cianuro y mercurio (Wolff, 2001).
} 
Es así como, antes del "evento Angosturas", el capital en torno a la minería ya había activado otros circuitos paralelos en tensión, que involucraban a actores del nivel global articulados exitosamente con el proyecto regional de institucionalidad estatal.

Estas relaciones cambian drásticamente en la década del 2000. Para ese momento, como se describió en la sección anterior, se venía intensificando en Santurbán la presencia de empresas de capital extranjero por medio de la compra de las empresas familiares, y la propuesta de exploración a cielo abierto en el páramo por parte de Greystar, en 2009, generó reacciones que hicieron visible, nacional e internacionalmente, la minería en la cuenca del río Suratá. En esta coyuntura, algunos habitantes de Bucaramanga se movilizaron bajo el lema "Agua sí, oro no", al tiempo que crearon organizaciones y se manifestaron públicamente, con gran incidencia en los medios de comunicación.

Parte de estas organizaciones se aglutinaron en el Comité por la Defensa del Agua y el Páramo de Santurbán (CDPAS), integrado por más de cuarenta organizaciones de la sociedad civil. Entre ellas se encuentran la Sociedad Santandereana de Ingenieros, la Asamblea Departamental, la Federación Nacional de Comerciantes (Fenalco), seccional Santander, y el Sindicato del Acueducto de Bucaramanga, además de representantes de la academia y ONG. Se trata, ante todo, de una alianza de intereses empresariales y profesionales urbanos enfocados en lo regional (oriente de Santander y suroccidente de Norte de Santander).

Este comité se conformó, según sus propios comunicados, con el objeto de trabajar por la defensa del agua y del medio ambiente, ante el proyecto de explotación minera que se pretendía adelantar en la estrella hídrica de Santurbán. Realizaron numerosas marchas, movilizaciones y foros de discusión ${ }^{17}$ con impactos regionales

\footnotetext{
17 Las fuentes consultadas reportan cuatro grandes marchas y tres foros y eventos académicos en la región durante el periodo estudiado.
} 
tanto en Santander como en Norte de Santander, ${ }^{18}$ a los que se hizo eco en medios de comunicación locales y nacionales.

Las organizaciones del CDAPS también establecieron vínculos de afinidad con organizaciones internacionales. El Comité, en asocio con la Asociación Interamericana por la Defensa del Ambiente (AIDA), el Centro International de Derecho Ambiental (CIEL) y Alerta Minera Canadá (MiningWatch Canada), presentó una queja contra el Fondo de Inversiones del Banco Mundial en relación con el proyecto Angostura, para exigir una auditoría a la inversión y el retiro del proyecto. Producto de esta acción, se generó un informe que recogía las preocupaciones de la sociedad civil, basado en entrevistas a representantes de diferentes sectores. Este informe fue publicado en junio de 2013, junto con la decisión de realizar una auditoría a la compra de acciones de la empresa EcoOro por parte del CFI, con el fin de establecer si este organismo consideró la evaluación de los riesgos sociales y ambientales antes de realizar la inversión.

La manera en que se configuró el movimiento ambiental antiminero en torno a Santurbán coincide con lo planteado por Damonte y Castillo (2012) acerca de los procesos de globalización que han facilitado la interrelación entre estos grupos de interés y actores sociales que, desde distintos puntos del globo, se entrelazan alrededor de las cadenas productivas generadas por la minería. A la luz de la política de la escala, el extractivismo catalizó la configuración de nuevas alianzas y de nuevos sujetos politicos, contribuyendo a consolidar la escala regional de control del territorio, centrada en actores urbanos de las capitales departamentales.

El análisis multiescalar y multitemporal de las relaciones en torno a la extracción del oro y la defensa del agua en Santurbán permite caracterizar este ambientalismo como un movimiento de origen urbano, estrechamente entretejido con proyectos políticos regionales, nacionales y globales y con escasos vínculos directos

\footnotetext{
18 También hubo reacciones y movilizaciones en Cúcuta y Pamplona, donde se realizaron eventos como el Foro sobre la conservación y preservación de los recursos hídricos y la biodiversidad de Norte de Santander en defensa del páramo de Santurbán, organizado por la organización estudiantil de la Universidad de Pamplona, en el que participaron representantes de la autoridad ambiental regional (Corponor), el Comité del Agua de Norte de Santander, estudiantes y docentes.
} 
con el territorio paramuno. En este caso, la defensa del agua emprendida por estos sectores se distancia, y cuestiona la actividad minera, incluyendo aquella ejercida por los habitantes de Vetas y California que, como se expresó anteriormente, tiene una trayectoria histórica de larga data. Como se verá a continuación, las manifestaciones públicas lideradas por este movimiento han tenido efectos concretos en el territorio, sus habitantes y los proyectos de extracción de oro.

\section{Áreas protegidas: ecos de la insurrección regional con su propia historia}

En 2014, después de dos años de reclamaciones de los movimientos ambientalistas contra la explotación del oro en Santurbán, la CDMB declaró el Parque Natural Regional (PNR) ${ }^{19}$ Páramo de Santurbán, en la unidad occidental. Esta declaratoria fue catalogada por algunos medios de comunicación como una victoria del ambientalismo.

Si bien es importante entender esta declaratoria en el contexto inmediato de la presión del ambientalismo civil regional - como una consecuencia de la insurrección regional frente al extractivismo-, es clave enfocarla también como parte de la historia de la institucionalidad ambiental colombiana y regional e inmersa en redes globales. Esta "insurrección" no habría sido efectiva si no se hubiera dado en un terreno que ya habían venido ganando proyectos conservacionistas nacionales y regionales, que por momentos han sido también anclas del capital global.

En el CPJSB, en el periodo de estudio, se venía trabajando, además de en torno al PNR Santurbán, en la jurisdicción de la CDMB, en la ampliación del PNR Sisavita, ${ }^{20}$ en la unidad nororiental del Complejo-jurisdicción de Corponor.

${ }^{19}$ Los Parques Naturales Regionales son, junto con los Parques Nacionales Naturales, las figuras más estrictas de conservación, en las que no se permite ningún tipo de actividad económica aparte del ecoturismo.

${ }^{20}$ Incluye áreas en páramo de los municipios de Pamplona, Mutiscua y Cucutilla, Arbloledas, Salazar de las Palmas Cáchira y Villacaro. 
La unidad nororiental abarca parte de veinte municipios de Norte de Santander y corresponde a las zonas altas de la zona hídrica de los ríos Catatumbo y Arauca. Aunque en esta unidad se desarrollan actividades agropecuarias (Cercapaz, 2012), es la zona del páramo con menor superficie transformada, con mayores pendientes y con mayor presencia de grandes propiedades. La mayor demanda de agua de esta zona proviene de la cuenca del río Zulia, que aporta agua al acueducto de Cúcuta (capital departamental) y soporta gran parte de las actividades productivas de la región, incluyendo la generación de energía eléctrica. Numerosos acueductos municipales y veredales de otras cuencas se benefician también del agua de esta vertiente.

El proceso de ampliación del PNR Sisavita involucra a Corponor, la Gobernación del Departamento, la Asamblea Departamental, los alcaldes y personeros de los municipios y el SENA, y cuenta con el apoyo de la cooperación alemana, a través del programa Cercapaz de GTZ. ${ }^{21}$ En 2012 se sumó el apoyo de la Fundación Bavaria, propiedad de la empresa SABMiller, para la socialización del proyecto en los municipios involucrados.

Si bien este vínculo con el capital global es notorio, las acciones estatales en torno a la conservación de los páramos, que incluyen la elaboración de estudios de estado actual y planes de manejo, declaratoria de áreas protegidas y delimitación del ecosistema, se han desarrollado en cumplimiento de la legislación ambiental nacional desde hace varias décadas. A lo largo de la segunda mitad del siglo XX se dio en Colombia un fortalecimiento de la institucionalidad ambiental: en este periodo se estableció el Instituto de Desarrollo de los Recursos Naturales Renovables (Inderena) (1968) y se expidió el Código Nacional de Recursos Naturales (1974); en la región se crearon las dos autoridades ambientales (CDMB en 1965 y Corponor en 1993). En 1998 se configuró la iniciativa de planificación y gestión Región Nororiental (ENOR GENOR), que reunió a

${ }^{21}$ La Deutsche Gesellschaft für Internationale Zusammenarbeit (GIZ) es el ente del gobierno de la República Federal de Alemania a cargo de la cooperación internacional para el desarrollo sostenible y de la educación internacional. GIZ hace parte de la Water Future's Partnership (www.water-futures.org). 
instituciones ambientales regionales y nacionales, y dio origen a acciones de conservación estatales vigentes actualmente, como el Sistema Departamental de Áreas Protegidas (Acuerdo 011 de 2004) y el mismo PNR Sisavita.

En particular en torno a los páramos, si bien la normatividad se había estado robusteciendo en general (Sarmiento y Zapata, 2016), vale la pena mencionar que en 2011 se expidió el Plan de Desarrollo del gobierno entrante, en el que se prohibió tajantemente cualquier actividad minera o agropecuaria en los páramos del país y se estableció la necesidad de delimitar estos ecosistemas como un paso hacia dicha prohibición. Aunque la declaratoria del PNR es un proceso diferente a la delimitación, esta acción se suma a las acciones estatales de conservación y resistencia al avance del extractivismo minero en páramos.

Este marco normativo y las decisiones que de allí se derivan, que implican limitaciones al uso productivo del páramo, se fundamentan en argumentos y definiciones científicas. Tal como argumentan Sarmiento y Zapata (2016), el reconocimiento científico de la biodiversidad y de los beneficios que los páramos ofrecen a la sociedad ha influido directamente en la expedición de normas para su conservación. ${ }^{22}$ El eje central de la argumentación, en el caso de estas áreas protegidas en Santurbán, es la preservación de los servicios ecosistémicos relacionados con el agua (Osejo y Ungar, 2015), consumida por habitantes urbanos y sectores económicos.

La declaratoria del PNR Santurbán generó reacciones adversas en diferentes actores sociales vinculados a actividades mineras y agropecuarias en Santurbán, incluyendo a habitantes de los municipios de la alta montaña, organizaciones de productores, empresas mineras familiares, empresas mineras transnacionales y administraciones municipales, que se manifestaron en audiencias públicas, asambleas, sesiones del Congreso de la República y medios de comunicación de difusión regional (Santander y Norte de Santander)

\footnotetext{
22 El discurso científico es también una fuerza global que incide en el proceso que analizamos aquí, cuyas formas de desplegarse y articularse con proyectos de construcción de nación, entre otros, sería importante analizar.
} 
y nacional. ${ }^{23}$ Los alcaldes de los municipios de Vetas y California manifestaron su preocupación frente a los impactos sociales de estas medidas en diferentes espacios de interlocución y medios de comunicación. Estas preocupaciones también fueron expresadas por empresas mineras locales de Vetas, como Trompetero, y otros sectores productivos de este municipio. La Asociación de Mineros Tradicionales de Vetas (Asomineros) manifestó su alarma frente al desempleo que se generaría y argumentó que fue el proyecto de explotación a cielo abierto de EcoOro el que desencadenó las restricciones ambientales a la minería. Los propietarios de las pocas empresas familiares que aún funcionan insisten en que la problemática de la contaminación del agua por la actividad minera viene siendo enfrentada de tiempo atrás por parte de las empresas locales. Algunos habitantes del páramo relataron cómo en los momentos más críticos de las protestas en Bucaramanga contra la minería en páramos había un ambiente de rechazo contra ellos en la ciudad.

En la vertiente oriental, en torno a la ampliación de Sisavita, a pesar de que Corponor le ha apostado a un proceso "multiactor", con extenso trabajo de campo y numerosas actividades de socialización, la iniciativa también ha generado algunas reacciones contrarias, sobre todo de propietarios en páramo preocupados por las limitaciones a sus actividades productivas. Al respecto es importante considerar los hallazgos de Cairo et al. (2014) y Von Bertrab Tamm (2010) referentes a los efectos sociales de la conservación. Este último argumenta que las acciones no consensuadas debilitan el tejido social. Como producto de esto, la conservación pierde legitimidad frente a la población local, lo que endurece las posturas y dificulta la búsqueda conjunta de soluciones.

Es así como las redes que emergieron en torno al ambientalismo, que en este caso catalizaron iniciativas estatales (regionales) de conservación en áreas protegidas, entraron en tensión con actores vinculados al páramo a través de fuertes lazos históricos, físicos y simbólicos (Buitrago, 2012).

${ }^{23}$ En la mesa de integración del río Suratá, conformada en el 2011, participaron mineros, dueños de tierras y de negocios de los municipios de Vetas, California y Suratá, quienes establecieron diálogos con la Gobernación y el Congreso de la República para hacer visible la afectación a las actividades productivas locales por la creación de áreas protegidas. 


\section{Conclusiones}

A través del relato de vínculos multiescalares y dinámicos que se entretejen con el territorio paramuno, es posible hacer una lectura de la problemática socioambiental en torno a Santurbán más allá de la dicotomía agua-oro. La noción de fricción (Tsing, 2005) ayuda a cualificar estos vínculos, lo que hace visible cómo los universales -vinculados tanto a la noción de ambientalismo como a la de extractivismo- se entretejen con las particularidades de un lugar con una historia minera y agropecuaria de más de cuatro siglos y una institucionalidad ambiental arraigada en el país y en la región. Se hace evidente cómo esos encuentros son heterogéneos y desiguales y llevan a nuevos arreglos de cultura y poder.

En particular en Santurbán es posible identificar de qué formas el páramo, el oro y el agua constituyen ejes de encuentros y tensiones en el territorio de redes globales de poder, capital y significado. El paisaje paramuno cambia o se mantiene en el tiempo respondiendo en parte a estas interacciones global-local, entretejidas con características biofísicas del territorio y herencias históricas. El territorio actual se puede leer en parte a la luz de esta perspectiva integrada: en las coberturas altamente transformadas del sur de Santurbán, que desde la Colonia ha surtido de alimentos a la minería en el occidente del complejo, agenciada desde entonces por intereses transnacionales; en las áreas sustraídas a la producción agropecuaria y minera, que reflejan acciones de las autoridades ambientales con un marco normativo propicio al menos desde la década de 1970 y que hoy se ve reforzado por el anclaje de intereses conservacionistas globales; y, por supuesto, en la dinámica minera, que desde la Colonia ha moldeado el territorio.

De cara a la gobernanza del páramo, los resultados llaman la atención sobre lo imbricado de los discursos e intereses transnacionales en el territorio. Subrayamos la necesidad de tener en cuenta la agencia del capital global, la complejidad de sus vínculos y la importancia de hacer explícito un debate sobre la capacidad del Estado de incidir en este nivel transnacional, máxime cuando se 
encuentra históricamente embebido en redes que lo involucran. También consideramos central avanzar en el camino emprendido por Buitrago (2012), en investigaciones desde categorias analíticas como lugar (Escobar, 2003), para hacer visible la experiencia de las localidades y las formas específicas de estar y construir el mundo, en el marco de múltiples transformaciones y fricciones. Esta mirada contribuiría a pensar la gobernanza del territorio partiendo de la inclusión de los paramunos y sus expectativas de vida.

\section{Referencias}

Alimonda, H. (2011). La naturaleza colonizada. Buenos Aires: CLACSO.

Blaikie, P. y Brookfield, H. (1987). Land degradation and society. En P. Blaikie y H. Brookfield (Eds.). London: Methuen.

Bodin, O. y Crona, B. (2009). The role of social networks in natural resource governance: What relational patterns make a difference? Global Environmental Change, 19(3), 366-374.

Buitrago, E. (2012). Entre el agua y el oro: tensiones y reconfiguraciones territoriales en el municipio de Vetas, Santander (Tesis de pregrado). Universidad Nacional de Colombia, Bogotá. Recuperado de http://www. bdigital.unal.edu.co/9059/1/emersonandresbuitragohernandez.2012.pdf

Cercapaz. (2012). Lectura Integral de Territorio sobre la zona de SanturbánSisavita, Departamento Norte de Santander. Informe descriptivo sobre el territorio que se ubica en la cuenca del río Zulia 2011-2012. Deutsche Gesellschaft für Internationale Zusammenarbeit (GIZ), Bogotá.

Cubillos, A. (2011). El proceso de transformación del páramo de guerrero por sistemas de ganadería bovina (1960-2010), con énfasis en politicas públicas (Tesis de maestria). Universidad Nacional de Colombia, Bogotá. Recuperado de http:/ / www.bdigital.unal.edu.co/50346/1/alexandercubillosg.2011.pdf 
Damonte, G. y Castillo, G. (2012). Presentación: una mirada antropológica a las industrias extractivas en los Andes. Anthropologica, 28(28), 5-19.

Del Cairo, C., Montenegro-Perini, I. y Vélez, J. S. (2015). Naturalezas, subjetividades y politicas ambientales en el Noroccidente amazónico: reflexiones metodológicas para el análisis de conflictos socioambientales. Boletin de Antropología, 29(48), 13-40.

Escobar, A. (2003). "Mundos y conocimientos de otro modo". El programa de investigación de modernidad/colonialidad latinoamericano. Tabula Rasa, (1), 51-86.

Falzon, M. A. (Ed.). (2012). Multi-sited ethnography: theory, praxis and locality in contemporary research. Londres: Ashgate Publishing.

Gil Granada, J. F. (2010). Formación de la empresa moderna en Antioquia: el caso de la minería del oro en el Valle de los Osos y Anorí, 1820-1850 (Tesis doctoral en Historia). Universidad de Antioquia, Medellín. Recuperado de http://200.24.17.74:8080/jspui/handle/fcsh/104

Göbel, B. y Ulloa, A. (Eds.). (2014). Extractivismo minero en Colombia y América Latina. Bogotá: Universidad Nacional de Colombia.

Gudynas, E. (1992). Los múltiples verdes del ambientalismo latinoamericano. Nueva Sociedad, (122), 104-115.

Marcus, G. (2001). Etnografía en/del sistema mundo. El surgimiento de la etnografía multilocal. Alteridades, 11(22), 111-127.

Martínez-Alier, J. (2005). El ecologismo de los pobres: conflictos ambientales y lenguajes de valoración. Barcelona: Icaria.

Neumann, R. P. (2009). Political ecology: theorizing scale. Progress in Human Geography, 33(3), 398-406.

Nieto, M., Zapata, J., Osejo, A. y Ungar, P. (2016). El cuidado de los páramos. Estrategias públicas, privadas y comunitarias. En M. F. Gómez, L. A. Moreno, 
G. I. Andrade y C. Rueda (Eds.), Biodiversidad 2015. Estado y tendencias de la biodiversidad continental de Colombia (ficha 305). Bogotá: Instituto Alexander von Humboldt.

Osejo, A. y Ungar, P. (2014). Caracterización de actores y sus relaciones en el páramo de Santurbán (Informe de investigación). Instituto de Investigación de Recursos Biológicos Alexander von Humboldt y Unión Europea, Bogotá.

Palacio, D. (2015). Redes, actores y gobernanza desde un enfoque relacional. En P. Ungar (Ed.), Hojas de ruta. Guías para el estudio socioecológico de la alta montaña en Colombia (tomo II). Bogotá: Instituto de Investigación de Recursos Biológicos Alexander von Humboldt.

Palacio, D. y Hurtado, R. (2005). Narrativas y redes de la gestión ambiental de los humedales de Bogotá. Revista Nómadas, 22, 140-151.

Palacio, D. y van der Hammen, M. C. (2007). Redes heterogéneas del patrimonio. Los casos del Centro Histórico y el humedal Córdoba en Bogotá. Redes Revista Hispana de Análisis de Redes Sociales, 13(1). Recuperado de http:/ / revista-redes.rediris.es

Riaño, P. F. P. (2015). La minería colonial en el páramo de Santurbán, el caso de Las Montuosas, Vetas y Páramo Rico. Boletín de Historia y Antigüedades, 101(859), 517-573.

Sarmiento, C. y Ungar, P. (Eds.). (2014). Aportes a la delimitación del páramo mediante la identificación de los limites inferiores del ecosistema a escala 1:25.000 y análisis del sistema social asociado al territorio: Complejo de Páramos Jurisdicciones - Santurbán - Berlín Departamentos de Santander y Norte de Santander. Bogotá: Instituto de Investigación de Recursos Biológicos Alexander von Humboldt.

Sarmiento, C. E. y Zapata, J. (2016). Instrumentos jurídicos para la protección de los páramos. Límites territoriales y jurídicos. En M. F. Gómez, L. A. Moreno, G. I. Andrade, y C. Rueda, C. (Eds.), Biodiversidad 2015. Estado y tendencias de la biodiversidad continental de Colombia (ficha 307). Bogotá: Instituto Alexander von Humboldt. 
Sletto, B. (2009). 'Indigenous people don't have boundaries': reborderings, fire management, and productions of authenticities in indigenous landscapes. Cultural Geographies, 16, 253-277.

Svampa, M. (2012). Consenso de los commodities, giro ecoterritorial y pensamiento crítico en América Latina. Observatorio Social de América Latina (OSAL), XIII(32), 15-39.

Swyngedouw, E. (2004). Scaled Geographies: Nature, Place, and the Politics of Scale. En E. Sheppard y R. B. McMaster (Eds.), Scale and Geographic Inquiry: Nature, Society, and Method (pp. 129-153). Massachusetts: Blackwell Publishing Ltd, Malden.

Tsing, A. L. (2005). Friction: An ethnography of global connection. Princenton: Princeton University Press.

Ungar, P., Osejo, A., Roldán, L. y Buitrago, E. (2014). Caracterización del sistema social asociado al territorio. En C. Sarmiento y P. Ungar (Eds.). Aportes a la delimitación del páramo mediante la identificación de los límites inferiores del ecosistema a escala 1:25.000 y análisis del sistema social asociado al territorio: Complejo de Páramos Jurisdicciones - Santurbán Berlín Departamentos de Santander y Norte de Santander (pp. 54-68). Bogotá: Instituto de Investigación de Recursos Biológicos Alexander von Humboldt.

Von Bertrab Tamm, A. (2010). Conflicto social alrededor de la conservación en la Reserva de la Biosfera de Los Tuxtlas: un análisis de intereses, posturas y consecuencias. Nueva antropología, 23(72), 55-80. Recuperado de http://www.scielo.org.mx/scielo.php?script=sci_arttext\&pid=S0185$06362010000100004 \& \operatorname{lng}=\mathrm{es} \& \operatorname{tlng}=\mathrm{es}$

Wolff, E. (2001). Proyecto río Suratá: líneas de acción para reducir contaminación proveniente de la pequeña minería aurífera en Vetas y California (Departamento de Santander, Colombia). En C. Canepa, F. Hurschke y G. Medina, Jornada Internacional sobre el Impacto Ambiental del Mercurio Utilizado por la Minería Aurifera Artesanal en Iberoamérica. Jornada organizada por CITEY y GAMA, Lima. 
Zapata, J. (2012). Análisis de la información disponible para el complejo jurisdicciones Santurbán y Berlín, relativa a los procesos de ordenamiento territorial y actividad minera (Informe de investigación). Instituto Alexander Von Humboldt, Bogotá.

Zimmerer, K. (2006). Multilevel Geographies of Seed Networks and Seed Use in Relation to Agrobiodiversity Conservation in the Andean Countries. En K. Zimmerer (Ed.), Globalization and New Geographies of Conservation (pp. 141-165). Chicago: The University of Chicago Press.

\section{Cómo citar este artículo}

Osejo, A. y Ungar, P. (2017). ¿Agua sí, oro no? Anclajes del extractivismo y el ambientalismo en el páramo de Santurbán. Universitas Humanística, 84, 143-166. https://doi.org/10.11144/Javeriana.uh84.ason 
